\title{
Correction to: Gingival phenotype assessment methods and classifications revisited: a preclinical study
}

\author{
Kai R. Fischer ${ }^{1}$ - Jasmin Büchel ${ }^{1}$ - Tiziano Testori ${ }^{2,3,4}$ - Giulio Rasperini ${ }^{2,4} \cdot$ Thomas Attin $^{5}$ • Patrick Schmidlin ${ }^{1}$ \\ Published online: 27 March 2021 \\ (C) Springer-Verlag GmbH Germany, part of Springer Nature 2021
}

\section{Correction to: Clinical Oral Investigations. https://doi.org/10.1007/s00784-021-03860-5}

After publication of this paper, the authors determined that affiliation 1 has an omitted information.

The correct affiliation should be:

1. Clinic of Conservative and Preventive Dentistry, Division of Periodontology and Peri-implant Diseases, Centre of Dental Medicine, University of Zurich, Zurich, Switzerland

The original article has been corrected.

Publisher's note Springer Nature remains neutral with regard to jurisdictional claims in published maps and institutional affiliations.

The online version of the original article can be found at https://doi.org/ $10.1007 / \mathrm{s} 00784-021-03860-5$

Kai R. Fischer

kai.fischer@zzm.uzh.ch

1 Clinic of Conservative and Preventive Dentistry, Division of Periodontology and Peri-implant Diseases, Centre of Dental Medicine, University of Zurich, Zurich, Switzerland

2 Department of Periodontics and Oral Medicine, School of Dentistry, University of Michigan, Ann Arbor, MI, USA

3 IRCCS Orthopedic Institute Galeazzi, Milan, Italy

4 Department of Biomedical, Surgical and Dental Sciences, University of Milan, Milan, Italy

5 Clinic of Conservative and Preventive Dentistry, Centre of Dental Medicine, University of Zurich, Zurich, Switzerland 